\title{
To be or not to be a compatible solute: Bioversatility of mannosylglycerate and glucosylglycerate
}

\author{
Nuno Empadinhas, Milton S. da Costa* \\ Department of Biochemistry, Center for Neuroscience and Cell Biology, University of Coimbra, 3004-517 Coimbra, Portugal
}

Received 30 April 2008

\begin{abstract}
Mannosylglycerate (MG) is an intracellular organic solute found in some red algae, and several thermophilic bacteria and hyperthermophilic archaea. Glucosylglycerate (GG) was identified at the reducing end of a polysaccharide from mycobacteria and in a free form in a very few mesophilic bacteria and halophilic archaea. MG has a genuine role in the osmoadaptation and possibly in thermal protection of many hyper/thermophilic bacteria and archaea, but its role in red algae, where it was identified long before hyperthermophiles were even known to exist, remains to be clarified. The GG-containing polysaccharide was initially detected in Mycobacterium phlei and found to regulate fatty acid synthesis. More recently, GG has been found to be a major compatible solute under salt stress and nitrogen starvation in a few microorganisms. This review summarizes the occurrence and physiology of MG accumulation, as well as the distribution of GG, as a free solute or associated with larger macromolecules. We also focus on the recently identified pathways for the synthesis of both molecules, which were elucidated by studying hyper/thermophilic MGaccumulating organisms. The blooming era of genomics has now allowed the detection of these genes in fungi and mosses, opening a research avenue that spans the three domains of life, into the role of these two sugar derivatives. (C) 2008 Elsevier GmbH. All rights reserved.
\end{abstract}

Keywords: Compatible solutes; Mannosylglycerate; Glucosylglycerate; Biosynthesis

\section{Introduction}

The term compatible solute reflects the ability of small molecular weight organic compounds to accumulate in cells in response to external increases in osmotic pressure without compromising cell physiology [5]. Dissolved salts commonly determine the amount of water available to cells and $\mathrm{NaCl}$, in particular, is a major cause of water stress in aquatic environments. The vast majority of microorganisms are capable of

\footnotetext{
*Corresponding author.

E-mail addresses: numenius@cnc.uc.pt (N. Empadinhas), milton@ci.uc.pt (M.S. da Costa).
}

responding, within intrinsic limits, to changes in the salt concentrations of the environment and many resort to the accumulation of compatible solutes to counterbalance the external decrease in water availability and consequent decrease in internal turgor pressure. Neutral zwitterionic compatible solutes such as trehalose, glycine betaine, ectoine and proline are frequent osmolytes in many mesophilic bacteria [11]. On the other hand, negatively charged organic solutes like di-myo-inositolphosphate and mannosylglycerate (MG) have been often identified in hyper/thermophilic bacteria and archaea [45]. However, some compatible solutes have crossed the boundaries of a role in osmoadaptation and play a broader range of biological functions $[11,12]$. It is 
currently believed that nature's economy has provided alternative functional and structural missions for compatible solutes. Trehalose, for example, is involved in the protection of cells against a plethora of different types of stress such as freezing and heat tolerance, in addition to the classical role in osmoadaptation [12]. Furthermore, trehalose is also a structural component of the cell walls of several bacteria [38].

In this review we will address some of the factors known to elicit the accumulation of MG and glucosylglycerate $(\mathrm{GG})$ in members of the three domains of life as well as their presence in larger macromolecules with apparently unrelated functions. We will also illustrate the diversity of pathways governing $\mathrm{MG}$ and $\mathrm{GG}$ biosynthesis as well as the genes and enzymes involved, and their distribution.

\section{Mannosylglycerate distribution and physiological roles}

\section{Mannosylglycerate in red algae}

The first report of the occurrence of a low molecular weight organic solute composed of mannose and glycerate, named digeneaside, which we designate as MG, was published by Colin and Augier in the red seaweed Polysiphonia fastigiata (Rhodophyceae) in 1939 [7]. Only later, was its structure established by Bouveng et al. [4]. Although MG has initially been found in members of the order Ceramiales, and considered a taxonomic marker for this order, it has recently been detected in other red algae of the orders Gelidiales and Gigartinales and its taxonomic significance has been refuted [30]. However, the concentration of MG does not always respond to increases in the salinity nor does it accumulate at levels suitable to contribute to the internal osmotic pressure of most species under salt stress, a role attributed to polyols, namely mannitol [29].

\section{Mannosylglycerate in thermophilic bacteria and archaea}

Mannosylglycerate was later identified in the thermophilic bacteria Thermus thermophilus and Rhodothermus marinus [37]. MG accumulates in the majority of strains of $T$. thermophilus, under salt stress but, in many cases, trehalose is the major compatible solute [1]. Trehalose has also been detected in many of the organisms that accumulate MG and a synergistic role for the two solutes was proposed by Santos and da Costa [45]. The construction of MG- and trehalose-negative T. thermophilus mutants and the concomitant response to osmotic stress led to the confirmation that MG is essential for low-level osmotic adjustment, while trehalose accumula- tion is required for growth at higher salt concentrations $[2,48]$. Mannosylglycerate also behaves as a compatible solute in $R$. marinus, where its concentration increases with the salinity of the medium, along with very low levels of glutamate and trehalose. Oddly, a neutral form of MG designated mannosylglyceramide (MGA) replaced MG at higher salinities [47]. Furthermore, MG was also implicated in the response of $R$. marinus to thermal stress [3]. Mannosylglycerate also accumulates in members of the genus Rubrobacter, which represent a deep-branching lineage of the phylum Actinobacteria [17]. These organisms are extremely $\gamma$-radiation resistant and the species $R$. xylanophilus (optimal growth temperature $60^{\circ} \mathrm{C}$ ) is the most thermophilic member of this genus that includes two additional species, $R$. radiotolerans and $R$. taiwanensis which also accumulate MG [6]. However, R. xylanophilus shows constitutive accumulation of MG and neither salt or thermal stress, nor the medium composition had significant effects on MG levels [17].

Mannosylglycerate has been identified in the hyperthermophilic archaea of the genera Pyrococcus, Thermococcus, Palaeococcus, Aeropyrum, Stetteria and in some strains of Archaeoglobus, where it accumulates concomitantly with the increasing levels of $\mathrm{NaCl}$ in the growth medium [46]. The apparent restrictedness of $\mathrm{MG}$ to thermophilic bacteria and hyperthermophilic archaea (Fig. 1) contributed to the hypothesis that MG is important in thermal resistance. However, several studies with hyperthermophilic archaea have shown that upon thermal stress, di-myo-inositol-phosphate (DIP) is the dominant intracellular organic solute [46]. Curiously, the hyperthermophilic archaeon Palaeococcus ferrophilus, which does not accumulate DIP, was found to accumulate high levels of MG both under salt or thermal stresses strengthening the hypothesis of a role for MG in thermal adaptation [36].

\section{Glucosylglycerate occurrence and biological functions}

\section{Glucosylglycerate as a compatible solute of bacteria and archaea}

The organic solute GG is a structural analogue of MG that was originally identified in the marine cyanobacterium Agmenellum quadruplicatum grown under nitrogen-limiting conditions [31]. GG was also recently shown to behave as a compatible solute in the $\gamma$-proteobacterium Erwinia chrysanthemi under combined salt stress and nitrogen-limiting conditions, replacing glutamate and glutamine, the major compatible solutes when abundant sources of nitrogen are present in the medium [22]. Therefore, it is possible that 


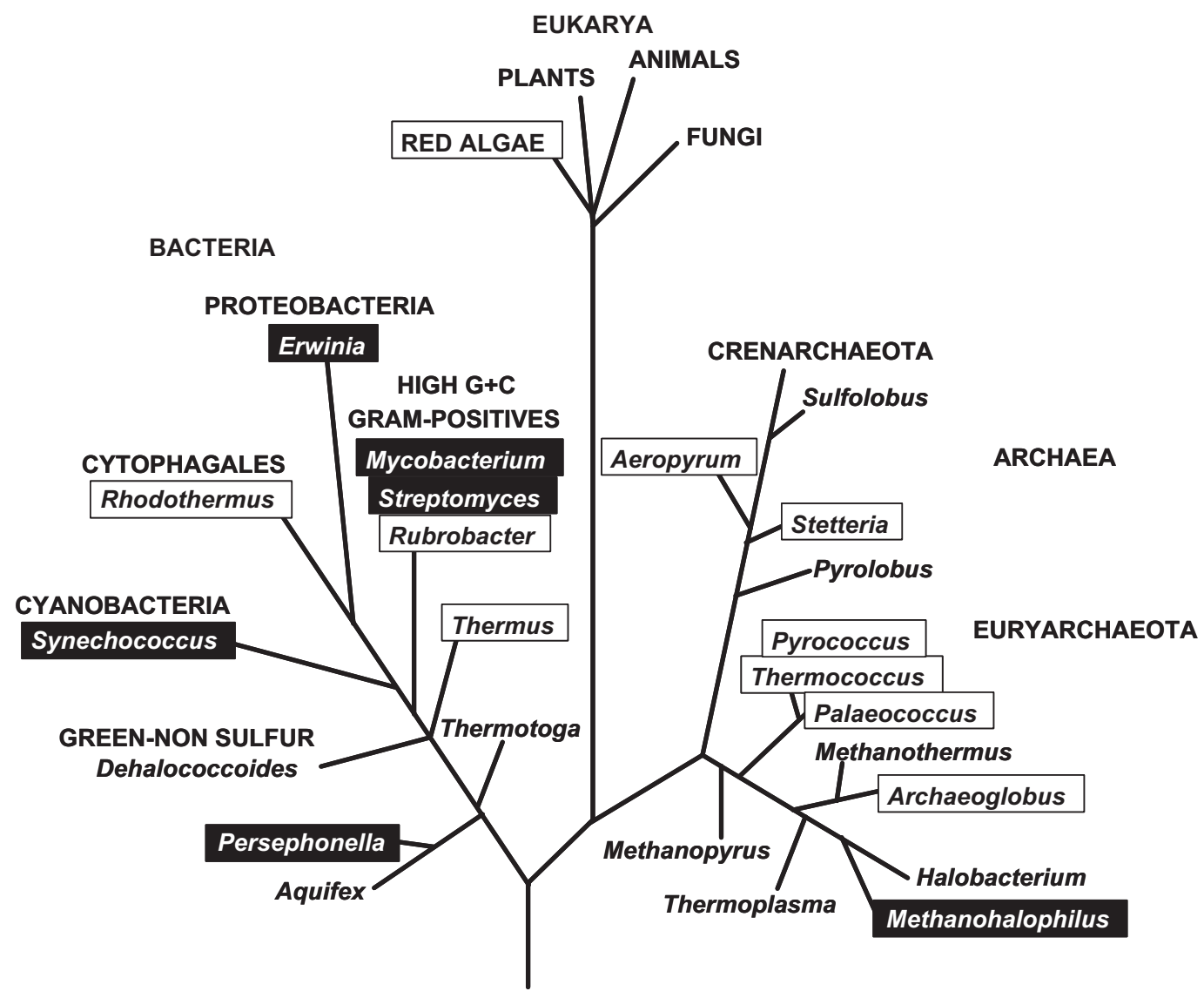

Fig. 1. Distribution of mannosylglycerate (white boxes) and glucosylglycerate (black boxes) in the three domains of life.

GG also accumulates during salt stress in other microorganisms where the corresponding genes have been detected, under conditions when limiting nitrogen has to be mobilized for the synthesis of other nitrogencontaining cell components [9].

Glucosylglycerate appeared to be a rare organic solute with a restricted distribution among mesophilic bacteria (Fig. 1) until it was unexpectedly identified in the thermophilic bacterium Persephonella marina, a member of the Aquificales, where it was suggested to act as a true compatible solute under salt stress [46]. This observation argued against the initial hypothesis of a restricted role for GG in organisms living at low temperatures and suggests that $\mathrm{GG}$ and $\mathrm{MG}$ could be functionally interchangeable in the adaptation to stress.

Glucosylglycerate has been detected in the halophilic archaeon Methanohalophilus portucalensis (Meth. portucalensis) as a minor component of the solutes pool, mostly dominated by amino acids and their derivatives [44]. Recent research on the biosynthesis of GG in the psychrotolerant archaeon Methanococcoides burtonii (M. burtonii) led to the hypothesis that GG could have a protective role when the organism grows under low nitrogen conditions. However, it was not possible to demonstrate this assumption since the organism did not grow in the absence of trimethylamine [8]. In spite of the occasional detection of free GG in a few organisms, the levels were too low to have any significant osmotic effect. Further research is required to clarify the role of GG that is suggested to be synthesized in the large numbers of organisms that, due to genome sequencing data, have been found to possess the appropriate gene homologues.

\section{The GG-containing methylglucose lipopolysaccharide (MGLP) from mycobacteria}

Free GG had been detected in trace amounts in Mycobacterium smegmatis and was considered to be the precursor for a GG-containing polysaccharide almost exclusively found in mycobacteria, designated methylglucose lipopolysaccharide [28,50]. This unusual polysaccharide contains about 16-20 hexoses some of which are methylated and have variable levels of acetate, propionate, isobutyrate and octanoate and succinic acid as lateral groups, while GG is located at the reducing end of the molecule [32,51]. The MGLP was found to sequester newly synthesized fatty acyl-CoAs, protecting them from cytoplasmatic esterases and decreasing 
inhibition by the product for fatty acid synthase I [26]. This polysaccharide can reach high intracellular concentrations $(0.5-1 \mathrm{mM})$, which allows mycobacteria to accumulate high levels of acyl-CoA derivatives without disturbing normal metabolism [21].

\section{The GG-containing glycolipid from Nocardia otitidis-caviarum}

Glucosylglycerate has also been found in the polar head of a glycolipid in Nocardia otitidis-caviarum [39]. This organism, which also synthesizes an MGLP similar to that of mycobacteria, is phylogenetically related to these organisms [40]. Other species of Nocardia have been examined where this glycolipid has not been found [39]. However, the physiological role of the GG-containing glycolipid is not known.

\section{Mannosylglycerate- and glucosylglycerate- derived compatible solutes}

MG is accumulated by $R$. marinus under moderate salt stress but replaced by the neutral derivative MGA when the organism is grown at salinities near the maximum tolerated (Fig. 2) [47]. Although it seems that MG is the substrate for an amidation reaction, the corresponding gene and enzyme as well as the regulatory events involved, have not been detected [3]. Curiously, the closely related $R$. obamensis can only produce and accumulate MG but not MGA [47].

A unique compatible solute derived from $G G$ has recently been identified in Petrotoga miotherma, a thermophilic member of the Thermotogales isolated from oil reservoirs, and characterized as mannosyl(1,2)-glucosylglycerate (MGG) (Fig. 2) [27]. The levels of MGG increase markedly with the $\mathrm{NaCl}$ concentration of the medium up to the optimum for growth. However, proline and $\alpha$-glutamate become the dominant compatible solutes at higher $\mathrm{NaCl}$ concentrations and the levels of MGG decrease. Curiously, the strategy for MGG production does not mirror that of $R$. marinus, which seems to convert a pre-existing compatible solute (MG) into a different form (MGA). In fact, GG has not been detected in P. miotherma in any of the conditions tested and the precursor for MGG biosynthesis has recently been identified to be glucosyl3-phosphoglycerate (GPG) (Fernandes, unpublished results). This compound is the phosphorylated precursor for the GG found in several organisms [8,9].

An additional GG derivative, glucosyl-(1,6)-glucosylglycerate (GGG) (Fig. 2) has been detected in P. marina, which is also known to accumulate GG [46]. This compound had already been detected in M. smegmatis where it was suspected to be one of the intermediates in the synthesis of the MGLP [28]. However, detailed information on the conditions leading to GG and GGG accumulation in $P$. marina is not available and the genes for GGG synthesis remain elusive.
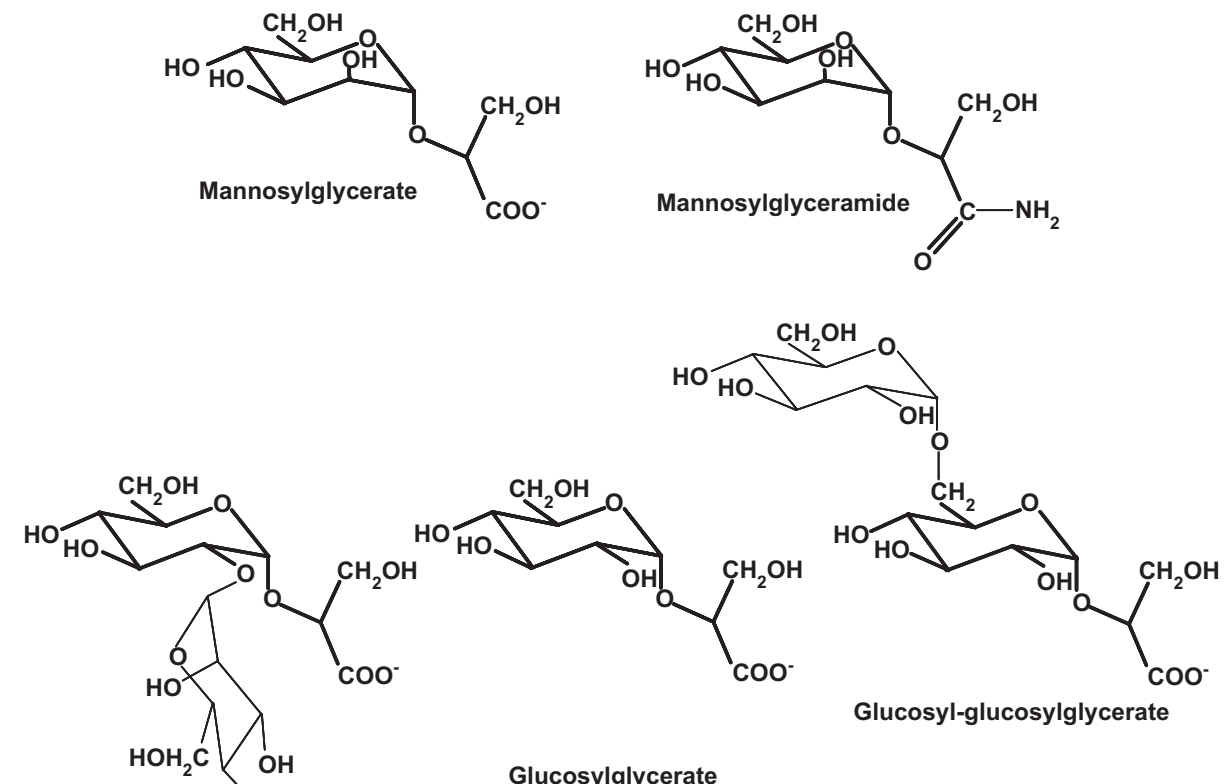

Mannosyl-glucosylglycerate

Fig. 2. Schematic representation of mannosylglycerate and glucosylglycerate and of the naturally occurring derivatives mannosylglyceramide, mannosyl-glucosylglycerate and glucosyl-glucosylglycerate $[27,46,47]$. 


\section{Biosynthetic pathways for MG}

\section{Mannosylglycerate biosynthesis in bacteria and archaea}

MG biosynthesis was initially investigated in the thermophilic bacterium $R$. marinus and found to involve a glycosyltransferase designated mannosylglycerate synthase (Mgs) that catalyzed the conversion of GDPmannose and D-glycerate into MG in one step [34] leading to the creation of the new glycosyltransferase family GT78 (www.cazy.org). However, an alternative pathway using 3-phosphoglycerate (3-PGA) instead of D-glycerate was also identified in this organism. The utilization of a non-phosphorylated acceptor has been observed for other sugar-based compatible solutes. Trehalose and sucrose, for example can be produced using glucose and fructose, respectively, as acceptor substrates [10,41]. The Mgs from $R$. marinus has been characterized and more recently the three-dimensional structure was determined [20,34].

Since one of the first archaeal genomes to be completely sequenced was that of Pyrococcus horikoshii, this organism was selected to study MG biosynthesis. MG synthesis in $P$. horikoshii cell-free extracts was tracked from GDP-mannose and 3-PGA through a phosphorylated intermediate, mannosyl-3-phosphoglycerate (MPG) [14]. This two-step strategy via a synthase and a phosphatase had frequently been observed for the synthesis of carbohydrate compatible solutes such as trehalose or glucosylglycerol [12,23]. The purification of the native mannosyl-3-phosphoglycerate synthase $(\mathrm{MpgS})$ allowed the identification of the corresponding gene (mpgS) in the P. horikoshii genome. The $m p g S$ gene, which was part of a 4 gene operon-like structure comprising two additional genes involved in GDPmannose synthesis, was functionally characterized as MpgS (EC 2.4.1.217) representing a new family of glycosyltransferases designated GT55 (www.cazy.org). A putative phosphatase gene, located immediately downstream $m p g S$, was functionally characterized as mannosyl-3-phosphoglycerate phosphatase (MpgP) (EC 3.1.3.70) [14].

The elucidation of the two-step pathway in hyperthermophilic archaea and the increasing genome sequences available allowed the detection of homologous genes, namely in a number of euryarchaeotes and in the crenarchaeotes Aeropyrum pernix and Staphylothermus marinus. Some crenarchaeal and euryarchaeal metagenomes isolated from environments such as deep-sea samples and forest soils were also found to contain $m p g S$ homologues, sometimes without the associated phosphatase gene $[24,42]$.

The two-step pathway was also identified and characterized in $R$. marinus and in $T$. thermophilus $[3,15]$. This pathway is identical to that found in hyperthermophilic archaea and involves the enzymes $\mathrm{MpgS}$ and $\mathrm{MpgP}$, which are also encoded by consecutive genes. Rhodothermus marinus is, so far, the only known organism to have two pathways for the synthesis of MG. Pathway multiplicity undeniably reflects a higher flexibility for the regulation of compatible solute pools upon different environmental stimuli, as shown in $R$. marinus, which differentially regulates each route when sensing salt or thermal stress; i.e. the single-step pathway is activated by thermal stress and the two-step pathway activation is related to salt stress [3].

Surprisingly, a gene for the synthesis of MG, comprising a $\mathrm{MpgS}$ and an MpgP in a single polypeptide, was identified in the mesophilic bacterium Dehalococcoides ethenogenes. This organism is known to completely dechlorinate groundwater pollutants such as tetrachloroethene (PCE) and trichloroethene, to ethene [35]. The functional expression of this gene (designated $m g s D$ ) both in E. coli and in Saccharomyces cerevisiae showed that the recombinant enzyme synthesized MG in vitro and in vivo, arguing in favor of a similar function in the native organism, which unfortunately is notoriously difficult to grow [16]. Preliminary experiments have shown that " $D$. ethenogenes" can grow at $\mathrm{NaCl}$ concentrations as high as $0.5 \mathrm{M}$ (Hsu and Zinder, unpublished results). Therefore, it is conceivable that MG may act as a compatible solute under salt stress in this organism. These data, along with the identification of MG genes in archaeal metagenomes retrieved from cold environments, refute the hypothetical exclusive association of MG with hyper/thermophilic prokaryotes.

\section{The ambiguous MpgS from Rubrobacter xylanophilus}

The synthesis of MG in $R$. xylanophilus was tracked in vivo from GDP-mannose and 3-PGA. The purification of the native enzyme allowed the identification of a highly divergent MpgS from which sequence homology to the known MpgSs had been extensively erased (Empadinhas et al., unpublished results). Adjacent to this mpgS gene we detected genes encoding two unspecific phosphatases, leading to the hypothesis that one or both could complete the two-step synthesis of MG. On the other hand, this atypical MpgS had high homology with one enzyme found in mycobacteria and related Actinobacteria, later found to be a glucosyl-3phosphoglycerate synthase (GpgS) with low homology to the homo-functional GpgSs from M. burtonii and $P$. marina $[8,9,18]$. In fact, the $R$. xylanophilus $\mathrm{MpgS}$ could synthesize GPG, the phosphorylated precursor of GG, with higher efficiency than that for MPG, although free GG has never been detected in $R$. xylanophilus. However, the failure to detect GG in $R$. xylanophilus 
does not assure that its precursor is not synthesized and incorporated into a macromolecule like the glycolipid found in $N$. otitidis-caviarum or the MGLP from mycobacteria [28,39]. In any case, the discovery of this ambiguous $\mathrm{MpgS} / \mathrm{GpgS}$ constituted a major breakthrough because it might represent the branch from which all known MpgSs and GpgSs evolved, indicating the possible link between the MG and the GG two-step pathways.

\section{MG genes in eukaryotes}

The biosynthesis of MG in red algae has not been addressed in detail, but recent sequences deposited in public databases (GenPept accession AAM93991) indicate that the single-step pathway (Mgs) found in $R$. marinus may be involved in the synthesis of $\mathrm{MG}$. The apparent acquisition of MG biosynthesis by red algae may reflect endosymbiosis of a prokaryote carrying a $m g s$ gene [33]. However, the rarity of sequences makes evolutionary conclusions premature and the origin and evolution of the Mgs pathway remains untraceable.

A functional mpgS is present in Magnaporthe grisea, as verified by its expression in $S$. cerevisiae and concomitant accumulation of this solute by the yeast [13]. However, the specific phosphatase gene normally associated with the $m p g S$ is absent from these organisms and the function of MPG is unknown. Moreover, $m p g S$ homologs have also been detected in the genomes of Neurospora crassa, Chaetomium globosum, Podospora anserina, Pyrenophora tritici-repentis and in Phaeosphaeria nodorum. Although polyols are the preferred compatible solutes in fungi, accumulation of $\mathrm{MG}$ cannot be entirely excluded.

Physcomitrella patens, the first moss (Bryophyta) whose genome was sequenced, has an intronless gene encoding a protein with high amino acid identity to the Mgs from R. marinus. Evolutionarily, P. patens symbolizes the first adaptation of ancient plants to land, and the exposure to drastic temperature fluctuations and water scarcity [43]. It is possible that this organism may accumulate MG as a trend acquired from red algae [49]. However, the factors underlying MG accumulation in red algae and possibly in mosses, as well as the evolutionary scenario, remain unknown.

\section{Pathways for the synthesis of GG}

\section{Glucosylglycerate biosynthesis in archaea and bacteria}

A mannosyl-3-phosphoglycerate phosphatase gene homologue $(m p g P)$ was found in the genome of $M$. burtonii, but a $m p g S$ gene was not detected.
A glucosyltransferase gene immediately upstream of the phosphatase was functionally characterized as a glucosyl-3-phosphoglycerate synthase (GpgS) [8]. The new family GT81 of glycosyltransferases was created to accommodate this enzyme and homologues (www. cazy.org). The enzyme from $M$. burtonii was specific for GDP-glucose and despite the similarity of this substrate with GDP-mannose and of the reaction mechanism, GpgSs and MpgSs shared no sequence identity, save for short motifs. Remarkably, this GpgS also had very low amino acid sequence identity with the homo-functional GpgSs from mycobacteria [18]. On the other hand, the recombinant MpgP from $M$. burtonii dephosphorylated GPG to GG and MPG to MG. Due to the vicinity with the $g p g S$ gene, it was designated $g p g P$. Similar flexibilities in substrate specificity were confirmed in vitro for the MpgPs from T. thermophilus, $P$. horikoshii, and "D. ethenogenes" [8].

The GpgS has homologues in many bacteria and a few archaea and was also partially identified in the first archaeon known to accumulate low amounts of GG, the halophilic Meth. portucalensis [8,44]. Interestingly, gpgS genes have been detected in the genomes of some extremely halophilic archaea such as Halorubrum lacusprofundi, Halobacterium sp., Natronomonas pharaonis and the square archaeon Haloquadratum walsbyi but not in other nearly 50 archaeal genomes sequenced so far. In archaea, the apparent restrictedness of gpgS homologues to extremely halophilic organisms still lacks physiological explanation.

The pathway for $\mathrm{GG}$ via $\mathrm{GpgS}$ and $\mathrm{GpgP}$ is mechanistically similar to the pathway for $\mathrm{MG}$ found in some hyper/thermophilic prokaryotes (see above). However, the genetic sequence of adjacent $m p g S / m p g P$ was not as conserved in $g p g S$ and $g p g P$ since they can be found in the genome as adjacent but divergently oriented genes, separate from each other or in operonlike structures [8]. The accumulation of significant levels of GG has only been shown to occur in Synechococcus sp. (formerly Agmenellum) and in E. chrysanthemii, despite the high number of $g p g S$ homologues found in bacteria $[22,31]$.

\section{Two pathways in a single operon for synthesis of GG in Persephonella marina}

Genes coding for $\mathrm{GpgS}$ and $\mathrm{GpgP}$ are contained in a complex operon-like structure in P. marina, along with a putative histidine kinase regulator, a putative glycerate kinase/dehydrogenase and a glucosyltransferase (Fig. 3) [9]. A second operon-like structure located immediately upstream contains genes related to phosphate uptake leading to the speculation that the synthesis of GG might be controlled by phosphate availability. The recombinant GpgS of $P$. marina has a more flexible 

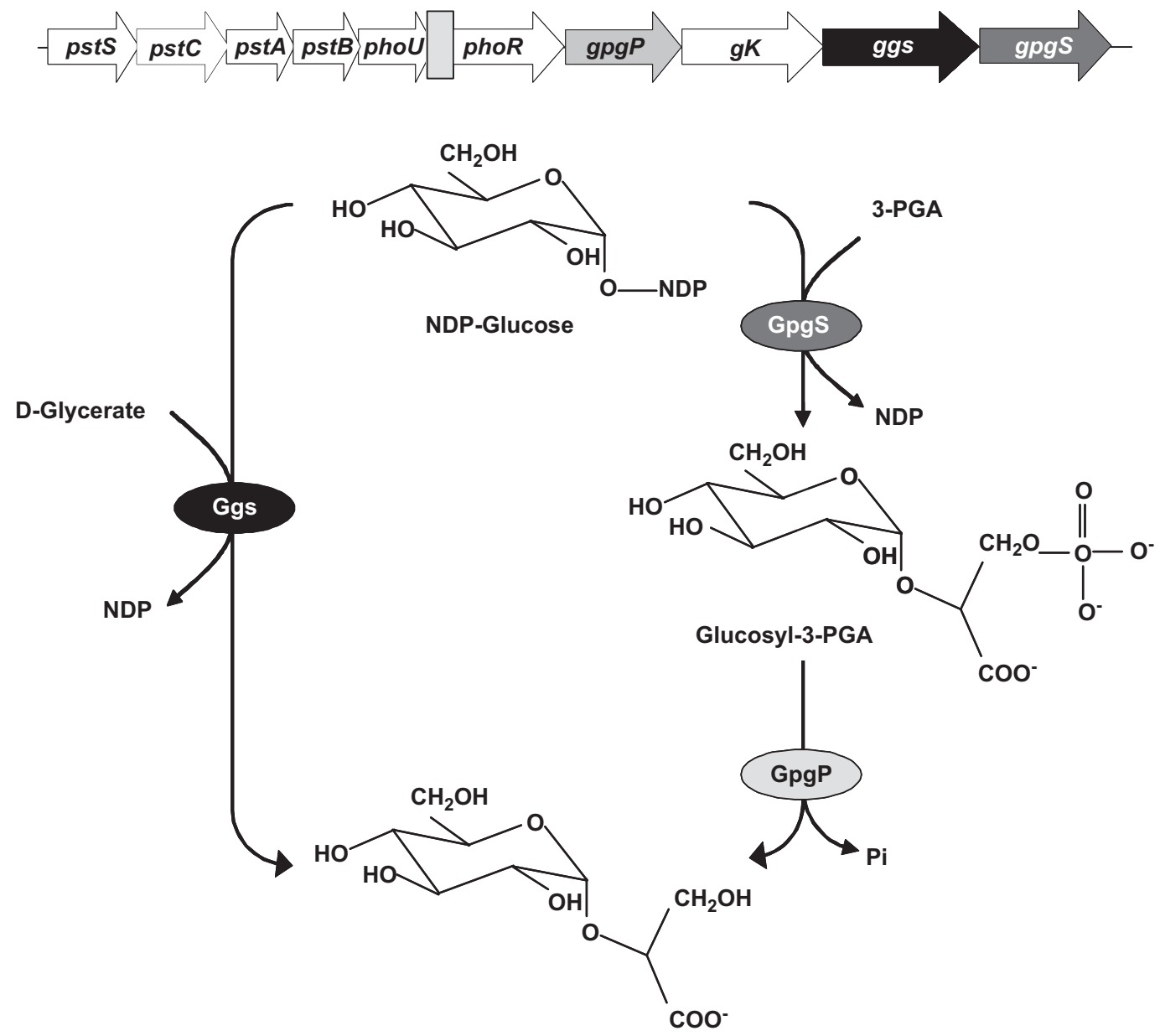

Glucosylglycerate

Fig. 3. Genetic organization and alternative pathways for synthesis of glucosylglycerate in Persephonella marina [9,19]. GpgP, glucosyl-3-phosphoglycerate phosphatase; Ggs, glucosylglycerate synthase; GpgS, glucosyl-3-phosphoglycerate synthase; gK, putative gycerate kinase; phoR, putative histidine kinase regulator; 3-PGA, 3-phosphoglycerate; NDP, nucleoside diphosphate. Genes upstream phoR encode a putative high-affinity Pi transporter system. The box indicates the putative promoter region for the GG operon.

substrate requirement than that of $M$. burtonii since it can synthesize GPG from any NDP-glucose donor and from 3-PGA. The recombinant GpgP, like the $M$. burtonii homologue, dephosphorylated GPG and MPG. The glycosyltransferase found in the abovementioned operon was found to catalyze the synthesis of GG from NDP-glucose and D-glycerate in one step, and was designated glucosylglycerate synthase (Ggs) [19]. The reaction catalyzed by this enzyme resembled that of the enzyme from the single-step pathway for MG described in R. marinus [34].

Putative Ggs sequences were detected in the genomes of several hyperthermophilic bacteria, archaea and also in some methanogens. To confirm its function, the putative $g g S$ gene from $P$. horikoshii has been expressed in $E$. coli and the recombinant product found to efficiently catalyze the synthesis of GG from ADPglucose and D-glycerate [13]. However, the function of this enzyme in the native organism is elusive since GG has never been detected in cell-free extracts. It is possible that GG is synthesized and incorporated into yet to be discovered larger molecules or that the growth conditions leading to GG synthesis in $P$. horikoshii and possible accumulation have not been found yet.

\section{The biosynthesis of GG in mycobacteria as precursor for the MGLP}

The identification of GG in the mycobacterial MGLP, as well as detection of homologues $(40 \%$ amino acid identity) to the $m p g S$ from $R$. xylanophilus in mycobacterial genomes, led to the study of the genes from two species from which the MGLPs have been studied, the fast-growing $M$. smegmatis and the slow-growing M. bovis $\mathrm{BCG}[18,28,50]$. The corresponding recombinant 
enzymes had GpgS activity in vitro. Interestingly, these GpgS sequences were only distantly related to the GpgSs found in M. burtonii, P. marina and homologues, and seemed to be restricted to some actinobacteria. The gpgS gene was present in all the mycobacterial genomes available and located in a conserved operon-like structure containing genes folP and $\operatorname{tag} A$, encoding a putative dihydropteroate synthase (DHPS) and a DNA glycosylase, respectively. Despite the fact that GG has been detected in $M$. smegmatis extracts no typical phosphatase gene (gpgP) has been detected in mycobacterial genomes suggesting that the dephosphorylation of GPG may reflect excess GPG that is not used for MGLP synthesis or is converted into GG by unspecific phosphatases/hydrolases [18].

\section{Biosynthesis of GG in Nocardia: a precursor for a glycolipid or for MGLP?}

The identification of a glycolipid in $N$. otitidiscaviarum in which the polar head was composed of GG esterified to aliphatic chains, extended the functions attributable to GG [39]. This organism also synthesizes MGLP and a gpgS of the mycobacterial type was detected in Nocardia farcinica, the only Nocardia species whose genome has been sequenced. The genetic organization of the $g p g S$-containing operon closely resembles that found in mycobacteria. The genome of Thermobifida fusca, another slightly thermophilic actinobacterium, contains two highly homologous and divergently oriented $\operatorname{gpg} S$ genes that seem to be the product of a recent duplication event [25]. It is not known if this organism synthesizes the MGLP, the GG-containing glycolipid or both, but it might be speculated that each gene is regulated differently and involved in different pathways, either for MGLP or glycolipid assembly.

\section{Concluding remarks}

Evidence gathered during the past few years points to a fundamental role of $\mathrm{MG}$ in osmotic adaptation of some organisms while others may also accumulate this compound to withstand hyperthermal conditions. However, the chemically related organic solute GG appears to have a limited role as a true compatible solute, although it was found to accumulate to significant amounts in a few organisms when the nitrogen supply was limited for growth at salt stress. Nevertheless, the genes coding for the key enzymes for the synthesis of this solute have a much broader distribution and further studies are necessary to confirm the functional role of GG as a compatible solute. However, GG has also found a structural role as it is present in larger structures such as a polysaccharide almost exclusively found in mycobacteria and in a glycolipid from a species of Nocardia. Future research will answer to the questions raised on the functional and structural roles for GG and MG in nature.

\section{Acknowledgments}

The authors wish to thank the many co-authors and collaborators who decisively contributed to our knowledge of compatible solute biology. We are especially grateful to Helena Santos and her group (ITQB, Oeiras, Portugal) for collaboration and helpful discussions on diversity and biosynthesis of compatible solutes from hyperthermophiles. This work was supported by Fundação para a Ciência e a Tecnologia, FCT, Portugal, and FEDER, Projects POCI/BIA-MIC/56511/2004 and A005/2005 Action V.5.1.

\section{References}

[1] S. Alarico, N. Empadinhas, C. Simões, Z. Silva, A. Henne, A. Mingote, H. Santos, M.S. da Costa, Distribution of genes for the synthesis of trehalose and mannosylglycerate in Thermus spp. and direct correlation with halotolerance, Appl. Environ. Microbiol. 71 (2005) 2460-2466.

[2] S. Alarico, N. Empadinhas, A. Mingote, C. Simoes, M.S. Santos, M.S. da Costa, Mannosylglycerate is essential for osmotic adjustment in Thermus thermophilus strains HB27 and RQ-1, Extremophiles 11 (2007) 833-840.

[3] N. Borges, J.D. Marugg, N. Empadinhas, M.S. da Costa, H. Santos, Specialized roles of the two pathways for the synthesis of mannosylglycerate in osmoadaptation and thermoadaptation of Rhodothermus marinus, J. Biol. Chem. 279 (2004) 9892-9898.

[4] H. Bouveng, B. Lindberg, B. Wickberg, Low-molecular carbohydrates in algae. Structure of the glyceric acid mannoside from red algae, Acta Chem. Scand. 9 (1955) 807-809.

[5] A.D. Brown, Microbial water stress, Bacteriol. Rev. 40 (1976) 803-846.

[6] L. Carreto, E. Moore, M.F. Nobre, R. Wait, P.W. Riley, R.J. Sharp, M.S. da Costa, Rubrobacter xylanophilus sp. nov., a new thermophilic species isolated from a thermally polluted effluent, Int. J. Syst. Bacteriol. 46 (1996) 460-465.

[7] H. Colin, J. Augier, Metabolism of Carbohydrates and Organic Acids in Plants (Exclusive of Bacteria and Fungi) Comptes Rendus Hebdomadaires des Seances del Academie des Sciences, Paris, vol. 209, 1939, p. 1450.

[8] J. Costa, N. Empadinhas, L. Gonçalves, P. Lamosa, H. Santos, M.S. da Costa, Characterization of the biosynthetic pathway of glucosylglycerate in the archaeon Methanococcoides burtonii, J. Bacteriol. 183 (2006) 1022-1030.

[9] J. Costa, N. Empadinhas, M.S. da Costa, Glucosylglycerate biosynthesis in the deepest lineage of the Bacteria: 
characterization of the thermophilic proteins $\mathrm{GpgS}$ and GpgP from Persephonella marina, J. Bacteriol. 189 (2007) 1648-1654.

[10] L. Curatti, A.C. Porchia, L. Herrera-Estrella, G.L. Salerno, A prokaryotic sucrose synthase gene (susA) isolated from a filamentous nitrogen-fixing cyanobacterium encodes a protein similar to those of plants, Planta 211 (2000) 729-735.

[11] M.S. da Costa, H. Santos, E.A. Galinski, An overview of the role and diversity of compatible solutes in Bacteria and Archaea, Adv. Biochem. Eng. Biotechnol. 61 (1998) 117-153.

[12] A.D. Elbein, Y.T. Pan, I. Pastuszak, D. Carroll, New insights on trehalose: a multifunctional molecule, Glycobiology 13 (2003) 17R-27R.

[13] N. Empadinhas, Pathways for the synthesis of mannosylglycerate in prokaryotes: genes, enzymes and evolutionary implications, Ph.D. Thesis, University of Coimbra, Portugal, 2005.

[14] N. Empadinhas, J.D. Marugg, N. Borges, H. Santos, M.S. da Costa, Pathway for the synthesis of mannosylglycerate in the hyperthermophilic archaeon Pyrococcus horikoshii. Biochemical and genetic characterization of key enzymes, J. Biol. Chem. 276 (2001) 43580-43588.

[15] N. Empadinhas, L. Albuquerque, A. Henne, H. Santos, M.S. da Costa, The bacterium Thermus thermophilus, like hyperthermophilic archaea, uses a two-step pathway for the synthesis of mannosylglycerate, Appl. Environ. Microbiol. 69 (2003) 3272-3279.

[16] N. Empadinhas, L. Albuquerque, J. Costa, S.H. Zinder, M.A.S. Santos, H. Santos, M.S. da Costa, A gene from the mesophilic bacterium Dehalococcoides ethenogenes encodes a novel mannosylglycerate synthase, J. Bacteriol. 186 (2004) 4075-4084.

[17] N. Empadinhas, V. Mendes, C. Simoes, M.S. Santos, A. Mingote, P. Lamosa, H. Santos, M.S. da Costa, Organic solutes in Rubrobacter xylanophilus: the first example of di-myo-inositol-phosphate in a thermophile, Extremophiles 11 (2007) 667-673.

[18] N. Empadinhas, L. Albuquerque, V. Mendes, S. MacedoRibeiro, M.S. da Costa, Identification of the mycobacterial glucosyl-3-phosphoglycerate synthase, FEMS Microbiol. Lett. 280 (2008) 195-202.

[19] C. Fernandes, N. Empadinhas, M.S. da Costa, Single-step pathway for synthesis of glucosylglycerate in Persephonella marina, J. Bacteriol. 189 (2007) 4014-4019.

[20] J. Flint, E. Taylor, M. Yang, D.N. Bolam, L.E. Tailford, C. Martinez-Fleites, E.J. Dodson, B.G. Davis, H.J. Gilbert, G.J. Davies, Structural dissection and highthroughput screening of mannosylglycerate synthase, Nat. Struct. Mol. Biol. 12 (2005) 608-614.

[21] L.S. Forsberg, A. Dell, D.J. Walton, C.E. Ballou, Revised structure for the 6-O-methylglucose polysaccharide of Mycobacterium smegmatis, J. Biol. Chem. 257 (1982) 3555-3563.

[22] R. Goude, S. Renaud, S. Bonnassie, T. Bernard, C. Blanco, Glutamine, glutamate, and $\alpha$-glucosylglycerate are the major osmotic solutes accumulated by Erwinia chrysanthemi strain 3937, Appl. Environ. Microbiol. 70 (2004) 6535-6541.
[23] M. Hagemann, U. Effmert, T. Kerstan, A. Schoor, N. Erdmann, Biochemical characterization of glucosylglycerol-phosphate synthase of Synechocystis sp. strain PCC 6803: comparison of crude, purified, and recombinant enzymes, Curr. Microbiol. 43 (2001) 278-283.

[24] S.J. Hallam, N. Putnam, C.M. Preston, J.C. Detter, D. Rokhsar, P.M. Richardson, E.F. DeLong, Reverse methanogenesis: testing the hypothesis with environmental genomics, Science 305 (2004) 1457-1462.

[25] S.D. Hooper, O.G. Berg, On the nature of gene innovation: duplication patterns in microbial genomes, Mol. Biol. Evol. 20 (2003) 945-954.

[26] M. Ilton, A.W. Jevans, E.D. McCarthy, D. Vance, H.B. White 3rd, K. Bloch, Fatty acid synthetase activity in Mycobacterium phlei: regulation by polysaccharides, Proc. Natl. Acad. Sci. USA 68 (1971) 87-91.

[27] C.D. Jorge, P. Lamosa, H. Santos, $\alpha$-D-Mannopyranosyl$(1 \rightarrow 2)$ - $\alpha$-D-glucopyranosyl- $(1 \rightarrow 2)$-glycerate in the thermophilic bacterium Petrotoga miotherma - structure, cellular content and function, FEBS J. 274 (2007) $3120-3127$

[28] K. Kamisango, A. Dell, C.E. Ballou, Biosynthesis of the mycobacterial $O$-methylglucose lipopolysaccharide. Characterization of putative intermediates in the initiation, elongation, and termination reactions, J. Biol. Chem. 262 (1987) 4580-4586.

[29] U. Karsten, J.A. West, Ecophysiological studies on six species of the Mangrove Red Algal genus Caloglossa, Aust. J. Plant Physiol. 20 (1993) 729-739.

[30] U. Karsten, S. Gors, A. Eggert, J.A. West, Trehalose, digeneaside, and floridoside in the Florideophyceae (Rhodophyta) - a reevaluation of its chemotaxonomic value, Phycologia 46 (2007) 143-150.

[31] V.H. Kollman, J.L. Hanners, R.E. London, E.G. Adame, T.E. Walker, Photosynthetic preparation and characterization of ${ }^{13} \mathrm{C}$-labeled carbohydrates in Agmenellum quadruplicatum, Carbohydr. Res. 73 (1979) 193-202.

[32] Y.C. Lee, C.E. Ballou, 6- $O$-Methyl-D-glucose from mycobacteria, J. Biol. Chem. 239 (1964) PC3602-PC3603.

[33] W. Martin, C. Rotte, M. Hoffmeister, U. Theissen, G. Gelius-Dietrich, S. Ahr, K. Henze, Early cell evolution, eukaryotes, anoxia, sulfide, oxygen, fungi first (?), and a tree of genomes revisited, IUBMB Life 55 (2003) 193-204.

[34] L.O. Martins, N. Empadinhas, J.D. Marugg, C. Miguel, C. Ferreira, M.S. da Costa, H. Santos, Biosynthesis of mannosylglycerate in the thermophilic bacterium Rhodothermus marinus. Biochemical and genetic characterization of a mannosylglycerate synthase, J. Biol. Chem. 274 (1999) 35407-35414.

[35] X. Maymo-Gatell, Y. Chien, J.M. Gossett, S.H. Zinder, Isolation of a bacterium that reductively dechlorinates tetrachloroethene to ethane, Science 276 (1997) $1568-1571$.

[36] C. Neves, M.S. da Costa, H. Santos, Compatible solutes of the hyperthermophile Palaeococcus ferrophilus: osmoadaptation and thermoadaptation in the order thermococcales, Appl. Environ. Microbiol. 71 (2005) 8091-8098.

[37] O.C. Nunes, C.M. Manaia, M.S. da Costa, H. Santos, Compatible solutes in the thermophilic bacteria 
Rhodothermus marinus and "Thermus thermophilus," Appl. Environ. Microbiol. 61 (1995) 2351-2357.

[38] M. Ohta, Y.T. Pan, R.A. Laine, A.D. Elbein, Trehalosebased oligosaccharides isolated from the cytoplasm of Mycobacterium smegmatis. Relation to trehalose-based oligosaccharides attached to lipid, Eur. J. Biochem. 269 (2002) 3142-3149.

[39] M.T. Pommier, G. Michel, Structure of $2^{\prime}, 3^{\prime}$-di- $O$-acyl- $\alpha$ D-glucopyranosyl $(1 \rightarrow 2)$-D-glyceric acid, a new glycolipid from Nocardia caviae, Eur. J. Biochem. 118 (1981) 329-333.

[40] M.T. Pommier, G. Michel, Isolation and characterization of an $O$-methylglucose-containing lipopolysaccharide produced by Nocardia otitidis-caviarum, J. Gen. Microbiol. 132 (1986) 2433-2441.

[41] Q. Qu, S.J. Lee, W. Boos, TreT, a novel trehalose glycosyltransferring synthase of the hyperthermophilic archaeon Thermococcus litoralis, J. Biol. Chem. 279 (2004) 47890-47897.

[42] A. Quaiser, T. Ochsenreiter, H.P. Klenk, A. Kletzin, A.H. Treusch, G. Meurer, J. Eck, C.W. Sensen, C. Schleper, First insight into the genome of an uncultivated crenarchaeote from soil, Environ. Microbiol. 4 (2002) 603-611.

[43] S.A. Rensing, D. Lang, A.D. Zimmer, A. Terry, et al., The Physcomitrella genome reveals evolutionary insights into the conquest of land by plants, Science 319 (2008) 64-69.

[44] D.E. Robertson, M. Lai, R.P. Gunsalus, M.F. Roberts, Composition, variation, and dynamics of major osmotic solutes in Methanohalophilus strain FDF1, Appl. Environ. Microbiol. 58 (1992) 2438-2443.
[45] H. Santos, M.S. da Costa, Compatible solutes of organisms that live in hot saline environments, Environ. Microbiol. 4 (2002) 501-509.

[46] H. Santos, P. Lamosa, N. Borges, T.Q. Faria, C. Neves, The physiological role, biosynthesis and mode of action of compatible solutes from (hyper)thermophiles, in: C. Gerday, N. Glandorff (Eds.), Physiology and Biochemistry of Extremophiles, ASM Press, Washington, DC, 2007, pp. 86-103.

[47] Z. Silva, N. Borges, L.O. Martins, R. Wait, M.S. da Costa, H. Santos, Combined effect of the growth temperature and salinity of the medium on the accumulation of compatible solutes by Rhodothermus marinus and Rhodothermus obamensis, Extremophiles 3 (1999) 163-172.

[48] Z. Silva, S. Alarico, A. Nobre, R. Horlacher, J. Marugg, W. Boos, A.I. Mingote, M.S. da Costa, Osmotic adaptation of Thermus thermophilus RQ-1: lesson from a mutant deficient in synthesis of trehalose, J. Bacteriol. 185 (2003) 5943-5952.

[49] J.W. Stiller, B.D. Hall, The origin of red algae: implications for plastid evolution, Proc. Natl. Acad. Sci. USA 94 (1997) 4520-4525.

[50] G. Tuffal, R. Albigot, M. Riviere, G. Puzo, Newly found 2- $N$-acetyl-2,6-dideoxy- $\beta$-glucopyranose containing methyl glucose polysaccharides in $M$. bovis BCG: revised structure of the mycobacterial methyl glucose lipopolysaccharides, Glycobiology 8 (1998) 675-684.

[51] K.K. Yabusaki, C.E. Ballou, Effect of polymethylpolysaccharides on the hydrolysis of palmitoyl coenzyme A by a thioesterase from Mycobacterium smegmatis, J. Biol. Chem. 254 (1979) 12314-12317. 